\title{
Black palm squirrel (Funambulus palmarum Linn.) from India: association with a frame shift mutation in the MC1R gene
}

\author{
R. Dileepkumar ${ }^{1,2, *}$, K. Anaswara ${ }^{1,2}$, V. Navya $^{2}$, S. Beena ${ }^{3}$, A. Jacob ${ }^{4}$, L. Divya ${ }^{5}$, \\ A. S. Vijayasree ${ }^{6}$, V. Deepthi ${ }^{2}$, G. Renganayaki ${ }^{2}$, P. R. Shidhi ${ }^{2}$, M. A. Akbarsha ${ }^{7}$, \\ K. P. Laladhas ${ }^{8}$, Achuthsankar S. Nair ${ }^{2}$, P. R. Sudhakaran ${ }^{2}$ and O. V. Oommen ${ }^{2, *}$ \\ ${ }^{1}$ Indriyam Biologics Private Limited, Sree Chitra Tirunal Institute for Medical Sciences and Technology, Thiruvananthapuram 695001 , India \\ ${ }^{2}$ Department of Computational Biology and Bioinformatics, University of Kerala, Thiruvananthapuram 695 581, India \\ ${ }^{3}$ OmicsGen Life Sciences Pvt Ltd, Cochin 682 301, India \\ ${ }^{4}$ Zoological Garden, Thiruvananthapuram 695 033, India \\ ${ }^{5}$ Department of Animal Sciences, Central University of Kerala, Kasaragod 671 316, India \\ ${ }^{6}$ Department of Zoology, Fatima Mata National College, Kollam 691 001, India \\ ${ }^{7}$ Department of Biotechnology, National College (Autonomous), Tiruchirappalli 620 001, India \\ ${ }^{8}$ St Stephen's College, Pathanapuram 689 695, India
}

This study shows that the dominant mutation of Extension locus in the recessive locus of the agouti Indian three-striped palm squirrel results in constituting an active or hyperactive receptor. This is not inhibited by the agouti antagonist or agouti signalling protein, resulting in melanism. To the best of our knowledge, there is no earlier report of a melanic variant (black) of a three-striped palm squirrel (Funambulus palmarum Linn.) from India. The colour change is due to mutation and is traced to melanocortin-1 receptor $(M C 1 R)$ gene, where it is proved to be a sequence alteration causing a frame shift in the Extension locus of the wild type. This would have probably caused the constitutive activation of MC1R.

Keywords: Agouti signalling protein, dominant mutation, Funambulus palmarum, melanism, melanocortin-1 receptor.

PigmentATION facilitates an animal to adapt for its survival in the wild, including signalling, protection and thermoregulation ${ }^{1}$. In addition, melanism also helps camouflage animals from their predators. Melanin is the principal pigment responsible for the colour of hair and fur. Melanocytes produce different types of melanins such as eumelanin (dark brown) and pheomelanin (pale red). Melanin synthesis is a complex biochemical process that starts from the amino acid tyrosine and its metabolite; dopa and several internal and external factors regulate its production ${ }^{2}$. More than 100 loci are associated with vertebrate pigmentation ${ }^{3}$, among which the Extension locus (E-locus) that encodes melanocortin 1 receptor

*For correspondence. (e-mail: dileepkamukumpuzha@gmail.com; oommenvo@gmail.com)
(MC1R), and agouti-locus (A-locus) that encodes agouti signalling protein (ASIP) are considered critical. In the grey squirrel, the hairs are black- and yellow-pigmented in the dorsal stripes. These banded hairs, known as agouti hairs, are common in wild animals, and give an overall appearance of camouflage. These bands of different colours are caused by pulses of ASIP expression during hair growth and the result is a strand of hairs with bands of pheomelanin and eumelanin. This can happen only if the MC1R functions as a switch, with $\alpha$-melanocyte stimulating hormone ( $\alpha$-MSH) binding as 'on' mode and ASIP binding as 'off' mode ${ }^{4}$. Polymorphism in MC1R and ASIP genes is related to the coat changing colour, as reported in the Arabian camel ${ }^{5}$. Mutation in the MC1R affects its fundamental ability to function as a switch, resulting in colour changes of the coat in many vertebrates.

Melanic variants of grey squirrels are common in North America and also in parts of Europe. The specimen used in the present study is an accidental discovery of a juvenile rodent identified to be a melanic variant of the palm squirrel from Thiruvananthapuram, Kerala, South India. To the best of our knowledge, there is no record of genetic cause of a melanistic form of the Indian threestriped palm squirrel, Funambulus palmarum, belonging to the family Sciuridae. This report of melanistic appearance of a single specimen proves that a mutation in the gene coding for MC1R is the cause for it. As computational studies offer insights into the receptor-ligand binding modes to the nearest possible precision, we adopted an in silico approach to analyse the sequence and structural features of the MC1R protein from $F$. palmarum. We also probed the ligand-binding affinity of the receptor against its agonist, $\alpha$-MSH, as well as its antagonist, ASIP, which could provide insights into the mechanism. 


\section{Materials and methods}

\section{Specimen identification}

The specimen was spotted by farm labourers in the premise of the Central Tuber Crops Research Institute campus at Thiruvananthapuram $\left(8^{\circ} 32^{\prime} 55.68^{\prime \prime} \mathrm{N}, 76^{\circ} 55^{\prime} 2.28^{\prime \prime} \mathrm{E}\right)$, during routine fieldwork. The animal was housed in a cage at home and at the Department of Zoology, University of Kerala, Thiruvananthapuram $\left(8^{\circ} 34^{\prime} 0^{\prime \prime} \mathrm{N}, 76^{\circ} 53^{\prime} 0^{\prime \prime} \mathrm{E}\right)$ after obtaining permission from the Kerala Forest Department (WLD/112/2008). The animal was sexed, fed properly and kept under observation for its behaviour during the study period. The identity of the juvenile rodent-like organism was confirmed by experts to be a variant of the threestriped palm squirrel. Three wild Indian three-striped palm squirrels were also caged separately as controls for the melanistic variant. Hairs from dorsum, flank and belly of both wild and melanic squirrels were examined in detail under a stereo-microscope (Olympus Magnus MSZ-TR Model, New Delhi, India). Blood samples were collected from the melanistic and wild squirrels, and genomic DNA was extracted using a commercial kit (Qiagen Tissue kit, Germany). The isolated DNA was sequenced for cytochrome oxidase subunit I (COI) and 16S rRNA, and analysed using nucleotide BLAST and CLUSTAL $\mathrm{W}$ programs to confirm the identity of the species. Next, we studied the genetics behind the coat colour variation of the melanistic form.

\section{MC1R sequencing}

The MC1R genes of both melanistic and wild-type squirrels were sequenced from the isolated DNA fractions. The sequences were inspected manually with special attention to heterozygotes and aligned using the CLUSTAL W.

\section{Sequence analysis of $M C 1 R$}

The nucleotide sequences of the wild squirrels as well as the melanic variant and their translated protein sequences were subjected to pairwise local alignment using BLAST ${ }^{6}$. The sequence homologues were identified using BLAST search against the non-redundant (nr) database of NCBI. The protein sequences were also screened against ExpasyProsite database using the ScanProsite tool to identify the presence of any protein signature. Multiple sequence alignments with 15 different sequences were performed using the CLUSTAL Omega program at EMBL-EBI. The phylogenetic tree was generated using default parameters and maximum likelihood algorithm was implemented in the program MEGA using the TamuraNei method ${ }^{7}$.

\section{Structure prediction of $M C 1 R$}

The sequence modelling was done by employing a combined $a b$ initio and threading strategy at the I-TASSER server $^{8}$, from Zhang lab (Cambridge, UK). The structural stability of the receptor models was analysed using structure analysis and verification server (SAVES) which employs five programs, namely PROCHECK $^{9}$, WHATCHECK ${ }^{10}$, Errat ${ }^{11}$, Verify $3 D^{12}$ and Prove ${ }^{13}$ to calculate different parameters determining the overall quality of a predicted 3D model. Energy minimization of the modelled structures was performed by the Steepest Descent method using GROMOS96 force field implemented in the Swiss PDB Viewer ${ }^{14}$. Dali pairwise server v3.1 (ref. 15) and TM-align tool comparison was done between the predicted 3D models of MC1R from wild specimen and its variant. The molecular surface (MS) area $\left(\AA^{2}\right)$ and van der Waals (VW) surface volume $\left(\AA^{3}\right)$ of the binding cavities of MC1R sequences were computed using CASTp program ${ }^{16}$.

\section{Structure-based ligand docking studies}

The 3D models of MC1R proteins from the wild specimen and the melanic variant were subjected to structurebased ligand interaction studies. The ligands selected for the study were $\alpha-\mathrm{MSH}$ and ASIP. The 3D structure of $\alpha$-MSH was obtained from the Protein Data Bank (PDB ID: 2IQP). The structure of ASIP was obtained by computational modelling of the sequence of human ASIP (UniProt ID: P42127). Docking studies were carried out using two computational resources. The ZDOCK program ${ }^{17}$ was incorporated in the Discovery studio suite from Accelrys Inc, USA, and the on-line server PatchDock was employed for the receptor-ligand interaction studies. The key residues present at the extracellular binding cavity of MC1R were identified by CastP. The best binding poses from PatchDock were refined using the FireDock program ${ }^{18}$. The global energy of the docked conformations was noted and further analysed. The best ranked pose with the least global energy in which the ligand occupied an extracellular binding cavity of MC1R was identified and further analysed.

\section{Results}

\section{Specimen identification and sequence analysis}

Microscopic examination of hairs removed from the flank, belly and the dark and light striped regions revealed that the wild striped agouti squirrel has eight distinct hair types in the tail region and two in the belly, as well as light and dark striped regions compared to the uniform black coloration of hairs in the melanistic squirrel. Figure 1 summarizes the hair types in each phenotype. The distinct 


\section{RESEARCH ARTICLES}

colour patterns of the hair types indicate different combinations of eumelanin and pheomelanin pigments responsible for the agouti pattern. This gives the wild squirrel an overall grizzled appearance with three stripes on the upper portion and light coat colouration on the belly portion. The melanistic variant has only a single hair type with complete eumelanin pigmentation throughout its body (Figure 2).

Taxonomic and molecular studies confirmed the squirrel to be a female melanistic variant. The amplified and sequenced COI and 16S rRNA of wild and the melanistic specimens indicated that both the squirrels belonged to the family Rodentia and confirmed them as F. palmarum.

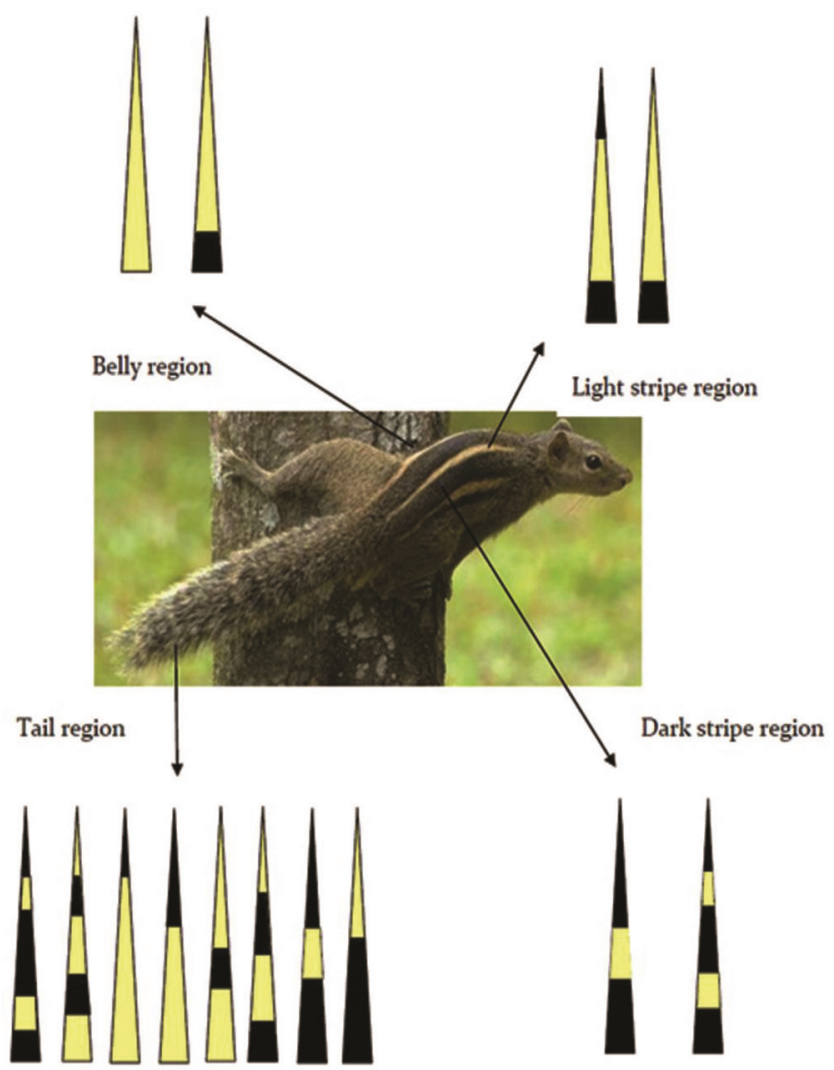

Figure 1. Diagrammatic representation of hair types from different parts of the body in wild type Funambulus palmarum.

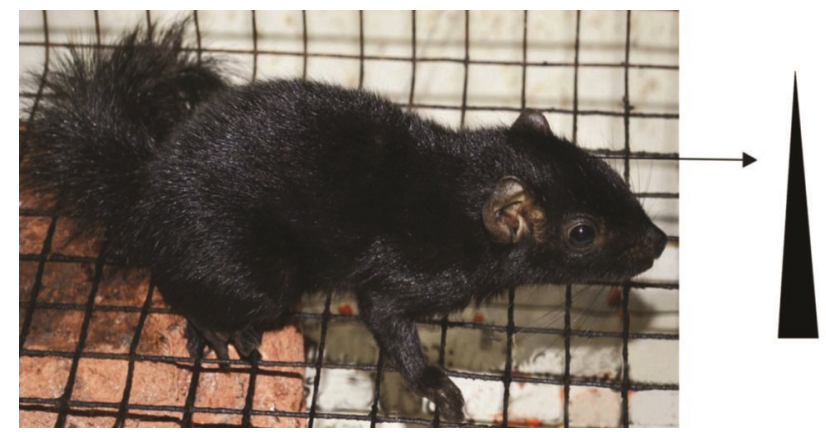

Figure 2. Diagrammatic representation of hair pattern in melanistic variant of $F$. palmarum.
Both COI (GU931775.1) and 16S rRNA (GU931776.1) sequences of the melanistic squirrel revealed more than $98 \%$ similarity with the wild-type squirrel. After species identification, the DNA samples were used for amplification and sequencing of the MC1R gene of about $948 \mathrm{bp}$ in the E-locus region and were submitted to NCBIGenBank (accession numbers KC149890.1 (wild type)

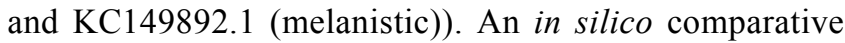
analysis of both these sequences revealed that the sequence along the entire length of the MC1R gene of wildtype squirrel was $948 \mathrm{bp}$, long and that of the melanistic squirrel was $939 \mathrm{bp}$ long. The corresponding translated proteins of 315 amino acid sequences in wild squirrel (GenBank accession no.: AGC10674.1) and 312 amino acids (GenBank accession no.: AGC10676.1) in the melanistic squirrel were also used for comparative studies. The protein sequences from both displayed the GPCR family 1 profile when screened against Expasy-Prosite database using the ScanProsite tool. The sequences differed in both the specimens (52-295), and the variant (48-80) at these positions. MC1R of the wild specimen had a 17 amino acid long consensus signature (SSLcFLGAIAIDRYIsI) of the GPCR family 1 in the position 127-143, which was missing in the variant. Insertion of the single base $T$ was at position 381 in the mutant melanistic, which was absent in all other species selected for analyses (Figure 3).

The phylogenetic relationships were inferred from maximum likelihood analyses of MC1R DNA sequences (Figure 4). Numbers above branches in the figure are support values from Bayesian analysis and non-parametric bootstrapping. The analysis and comparison of protein sequences revealed that the insertional mutation (of base $\mathrm{T}$ ) at the position 381 of the MC1R gene causes a shift in the reading frame (frame-shift mutation) of the protein at position 127 in the variant. Figure 5 depicts a close examination of the nucleotide sequence near the insertion site (from position 361 to 405 ) in F. palmarum and the variant along with the translated frame.

\section{Three-dimensional structure prediction and analysis}

As no suitable templates could be identified, a combined threading and ab initio method was adopted to model the structure of MC1R. The best predicted theoretical model of MC1R protein of the wild specimen had an optimum $C$-score of -0.24 , TM-score of $0.68 \pm 0.12$ and root mean square deviation (RMSD) value of $6.8 \pm 4.1 \AA$, whereas those of the variant were $-2.31,0.44 \pm 0.14$ and $11.7 \pm 4.5 \AA$ respectively (Figure 6 ). Structure optimization studies were carried out and the models were verified for their accuracy and stability: $84.7 \%$ of the amino acids in MC1R of $F$. palmarum were in the core regions of the Ramachandran plot in PROCHECK, and the ERRAT program quality factor obtained was 83.71. However, $85.5 \%$ of the residues were in the core regions of the 


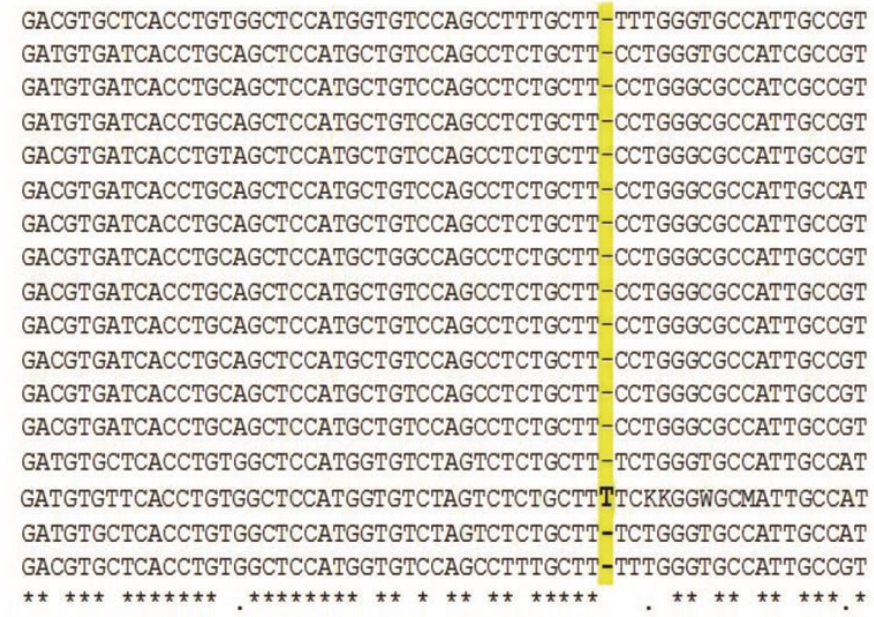

Figure 3. Multiple sequence alignment showing the insertion of base $\mathrm{T}$ at position 381 .

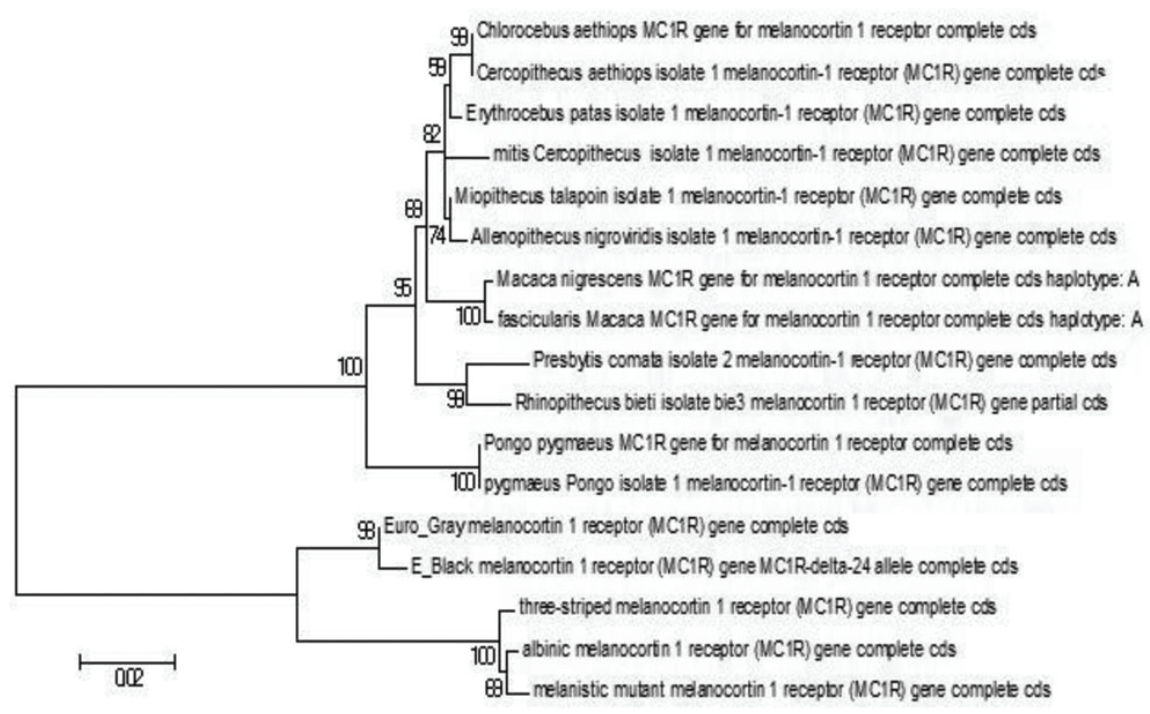

Figure 4. Maximum-likelihood phylogenetic tree of melanocortin-1 receptor (MC1R) sequences.

(a) 361 atg $\mid$ gtg $\mid$ tct $\mid$ agt $\mid$ ctc $\mid$ tgc|ttt $\mid$ ctg $\mid$ ggt $\mid$ gcc $\mid$ att $\mid$ gcc|ata $\mid$ gac $\mid$ cgc 405 $\begin{array}{llllllllllllllll}\text { M } & \text { V } & \text { S } & \text { S } & \text { L } & \text { C } & \text { F } & \text { L } & \text { G } & \text { A } & \text { I } & \text { A } & \text { I } & \mathbf{D} & \mathbf{R}\end{array}$

(b) 361 atg $\mid$ gtg $\mid$ tct $\mid$ agt $\mid$ ctc $|\operatorname{tgc}|$ ttt $|\operatorname{tct}| \operatorname{ggg}|\operatorname{tgc}|$ cat $|\operatorname{tgc}|$ cat $\mid$ aga $\mid \operatorname{ccg} 405$

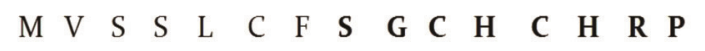

Figure 5. Reading frame analysis of MC1R sequences. $\boldsymbol{a}$, Reading frame of MC1R in wild type. $\boldsymbol{b}$, Shift in reading frame caused by the insertion of base $\mathrm{T}$ at position 381 of MC1R in the melanistic variant.

Ramachandran plot, and the quality factor obtained in ERRAT was 78.87 for the MC1R in the melanistic form when analysed for stereochemical stability in SAV server. MC1R protein receptor structures of the wild and melanistic specimens were structurally aligned based on the C-alphas with RMSD of $3.4 \AA$. The superimposition of the structures in TM-align tool provided a TM score of 0.69 , suggesting that both the proteins share a common SCOP/CATH fold (Figure 7).
These results confirm that the protein structures share similar features of protein members of the GPCR family. PDB ID: 4EIY was structurally most similar to the MC1R model of the wild specimen with an RMSD of $2.2 \AA$ and $Z$-score of 29.9. MC1R structure from the variant showed a slightly higher RMSD of 5.4, but with a good $Z$-score of 21.0. CASTp revealed the putative binding pockets in the extracellular surface of the MC1R protein from both the wild and melanistic squirrels. The residue contributions in each of these pockets were also noted (Figure 8).

\section{Molecular interaction studies}

The computational docking studies revealed that the agonist, $\alpha$-MSH, and the antagonist, ASIP, interacted in the extracellular pockets of MC1R in both the wild specimen and the variant (Figure 9). The competitive mode of binding of the ligands was evident from the binding energy as well as the ZDOCK score (Table 1): $\alpha$-MSH showed a 


\section{RESEARCH ARTICLES}

binding energy of $-29.02 \mathrm{Kcal} / \mathrm{mol}$ and ZDOCK score of 12.56 with the MC1R (wild specimen), whereas for ASIP it was $-31.34 \mathrm{kcal} / \mathrm{mol}$ and ZDOCK score of 13.26 . In the variant, $\alpha$-MSH interacted with MC1R with a comparable binding energy of $-28.14 \mathrm{kcal} / \mathrm{mol}$ and ZDOCK score of 12.08, with no favourable binding for ASIP.

It was observed that $\alpha$-MSH binds to the residues present in the $\mathrm{N}$-terminal domain and the extracellular loop 3 of MC1R in the wild squirrel. The key residues identified were Leu23, Pro265, Gln266, His267, Pro268 and Cys272. In the variant, $\alpha$-MSH binds strongly with the residues present at the N-terminal domain, EC loop 1, loop 2 and loop 3 with comparable binding energy. The interacting residues were Gln27, Leu94, Glu95, Pro178, Gly180, Pro182, Ala183 and Pro261. Interestingly, ASIP formed a strong linkage with MC1R (wild squirrel) through a disulphide bond between the residues Cys 272 and Cys132, which highlights the importance of the
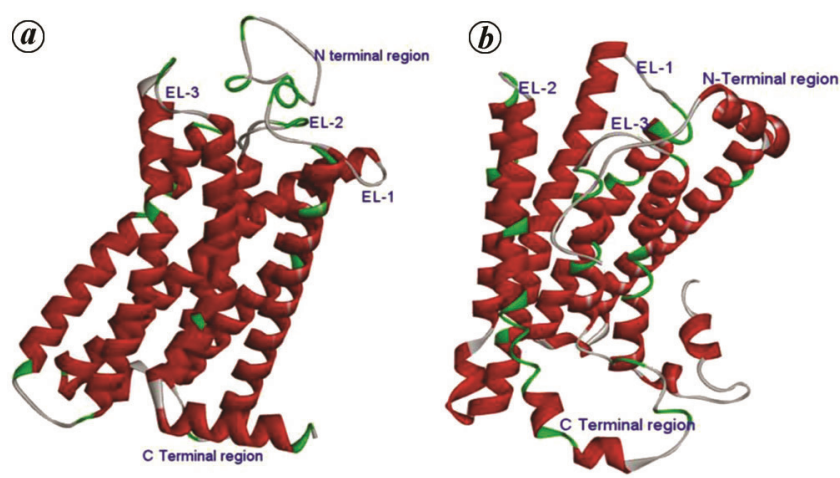

Figure 6. Visualization of the theoretical models of MC1R. $\boldsymbol{a}$, Wild type $F$. palmarum model. $\boldsymbol{b}$, Melanistic $F$. palmarum model.

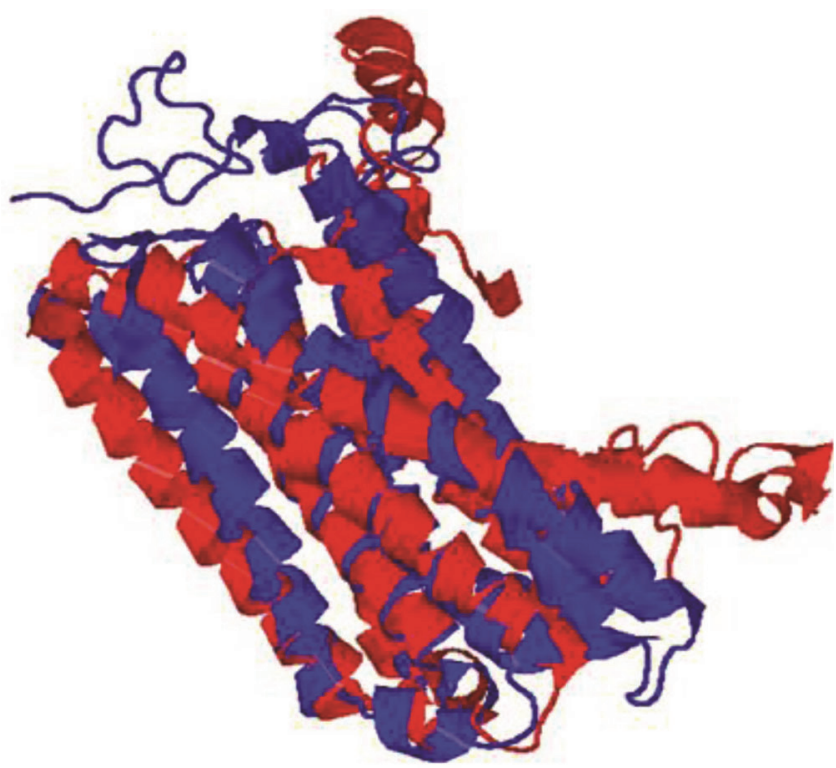

Figure 7. MC1R protein structure superimposition. Structure of wild squirrel (blue) superimposed on melanic squirrel (red). cysteine-rich C-terminal domain of ASIP in the complex formation with MC1R. ASIP did not bind effectively at the extracellular regions of the melanic squirrel (Figure 10). The Cys residue at position 272 in wild squirrel MC1R was actively binding both the agonist and antagonist, whereas in the melanistic variant Cys272 it was inactive. The $3 \mathrm{D}$ structure-based studies suggest that the frame shift mutation in the MC1R gene level resulted in an altered protein product which retains the binding affinity with the agonist, but restricts binding of the antagonist, promoting melanism.

\section{Discussion}

The E-locus is epistatic to the Agouti gene in the family Rodentia; hence, any mutation in the $E$-locus has a direct effect on coat coloration. Dominant mutations at MC1R, encoding constitutively active receptors, are not inhibited by the agouti antagonist in mouse and animals with dominant alleles of both loci which remain darkly pigmented $^{19}$. High-resolution atomic structures of MC1R are not yet available for $F$. palmarum. We perceived that furnishing reliable structural data for MC1R can contribute more to our understanding of the biological mechanism, behind its role in regulating melanism in F. palmarum. The molecular structure-prediction studies provide a prototypical report of the trans-membrane structural features of MC1R in F. palmarum and its variant. The 3D structure of MC1R from the wild specimen retains the topology of a typical GPCR, whereas MC1R from the melanistic individual deviates slightly from the topology. MC1R from wild specimen showed $96 \%$ identity with Sciurus carolinensis, and 12 amino acid changes between the two sequences in which six amino acids are substitutions of functionally similar ones. The changes were T14P, M16T, R22Q, Q27K, A89V, T93A, V137I, I170V, V183A, R220Q and I262V. MC1R protein sequence of European black squirrel is characterized by a deletion of the region 'SNALETTI' at the position 87-94 (ref. 4). The loss of glutamic acid in MC1R of European black squirrel leads to constitutive activation or hyperactivity of the receptor, resulting in eumelanogenesis and melanism ${ }^{3}$.

The mechanism underlying melanism in the Indian palm squirrel is perhaps different from that in the European black squirrel, since our comparative study shows that the region corresponding to positions 87-94 is 'SNVLETAI' in both the wild type and melanistic squirrels, and it retains the glutamic acid. The $3 \mathrm{D}$ structure comparison studies revealed biologically interesting similarities, thereby providing meaningful insights into the functional mechanism which may not be detected by sequence comparison. The RMSD values for the structures with homologs in PDB determine the extent of structural similarity between proteins. The results show that the C-terminal loop of ASIP is critical in binding 
Table 1. Molecular docking of agonist and antagonist with the receptor protein melanocortin-1 receptor (MC1R) from wild and melanistic squirrels

\begin{tabular}{llccc}
\hline Receptor protein & Ligand & $\begin{array}{c}\text { ZDOCK } \\
\text { score }\end{array}$ & $\begin{array}{c}\text { No. of favourable } \\
\text { interactions }\end{array}$ & $\begin{array}{c}\text { Global energy } \\
\text { (kcal/mol; FireDock) }\end{array}$ \\
\hline MC1R (wild) & $\alpha$-MSH & 12.56 & 14 & -29.02 \\
& ASIP & 13.26 & 19 & -31.34 \\
MC1R (melanic) & $\alpha$-MSH & 12.08 & 23 & -28.14 \\
& ASIP & \multicolumn{2}{c}{ No interaction in the extracellular cavity } \\
\hline
\end{tabular}

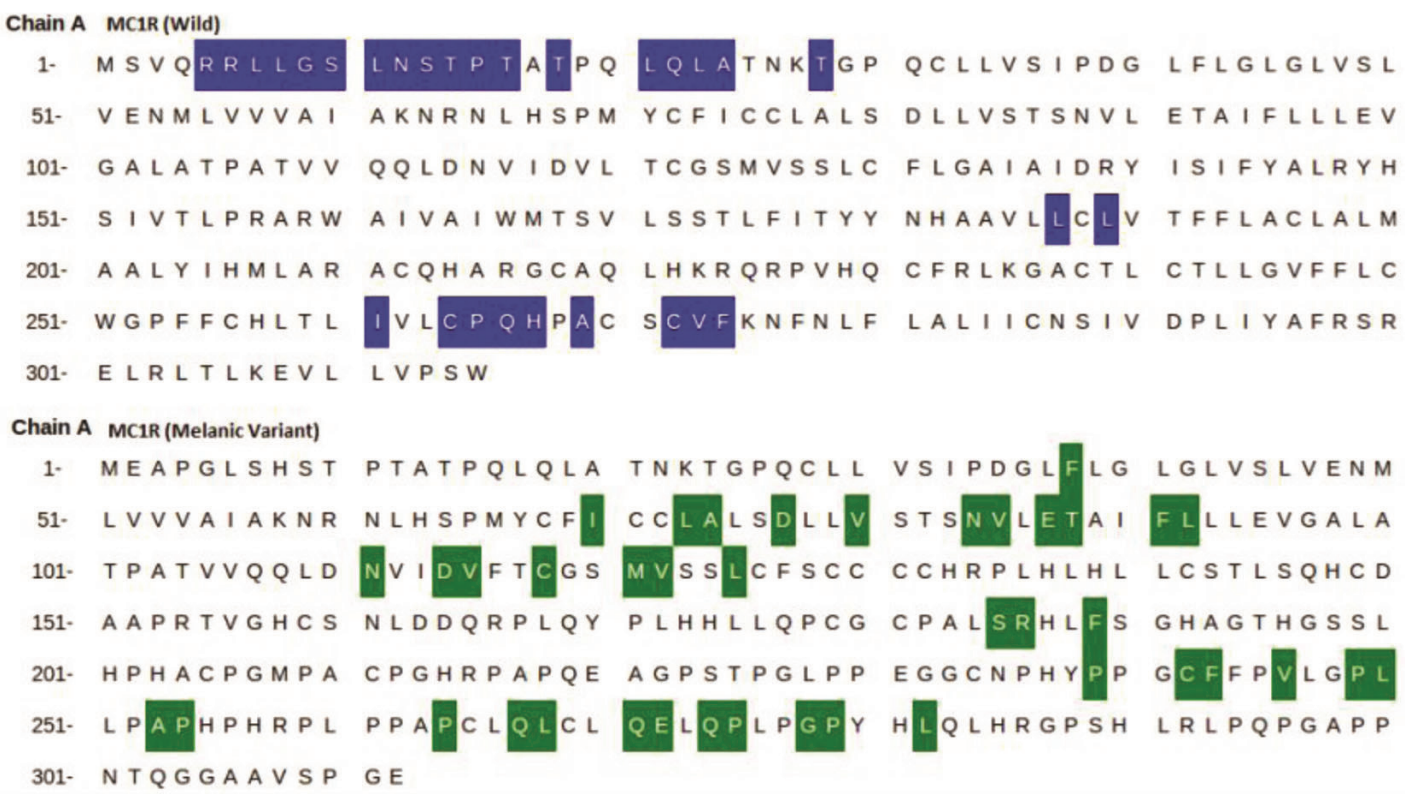

Figure 8. Residues involved in the extracellular binding pocket region of MC1R of wild type F. palmarum and melanistic F. palmarum.
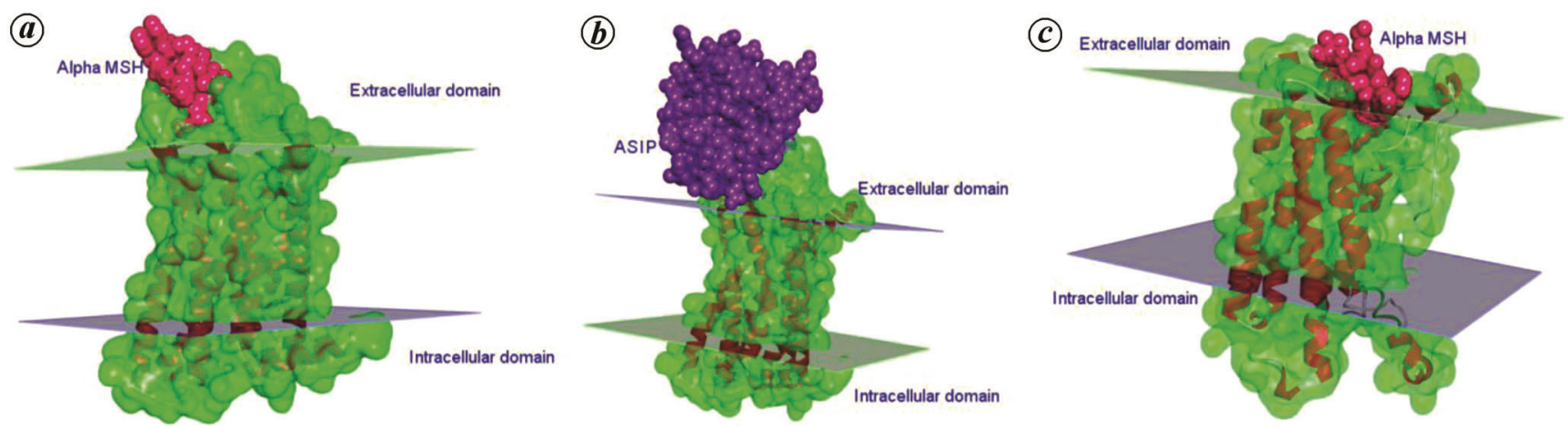

Figure 9. Comparative binding modes of MC1R against ligands. Binding of (a) agonist $\alpha$-MSH with MC1R (wild type); (b) $\alpha$-MSH with MC1R (melanistic variant) and (c) antagonist ASIP with MC1R (wild type).

with MC1R, which is in accordance with the study on loop swapped with the chimeras of agouti-related protein (AgRP) and ASIP against MC1R (ref. 20).

It is reported that the Cys-rich C-terminal domain of ASIP is responsible for melanocortin receptor binding activity in vitro ${ }^{21}$. In the present study, we use an in silico approach to analyse the sequence and structure of MC1R protein of $F$. palmarum and its melanistic variant. The insertion of a single base $\mathrm{T}$ at position 381 of the nucleotide sequence of MC1R in the melanistic squirrel causes a frame shift during translation, thereby altering the code for the structure of the protein. The change in conformation causes a change in binding of the cavity and, hence differential affinity towards the agonist $\alpha$-MSH and the antagonist ASIP. Binding of the agonist $\alpha$-MSH with MC1R is stronger in the melanistic squirrel than in the wild (agouti) squirrel, whereas ASIP has poor or little binding affinity with the melanistic variant of MC1R compared with that of wild squirrel. 
(a)

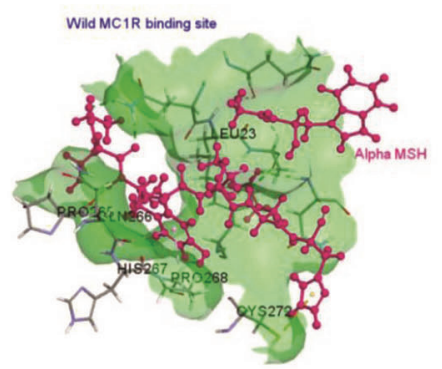

(b)

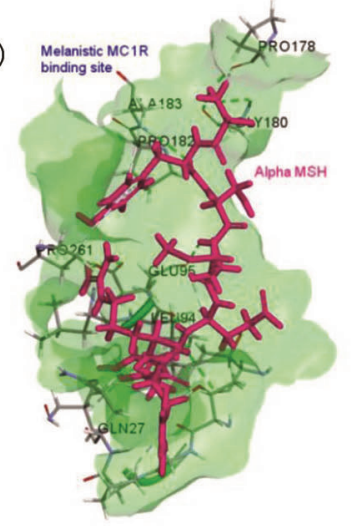

(c)

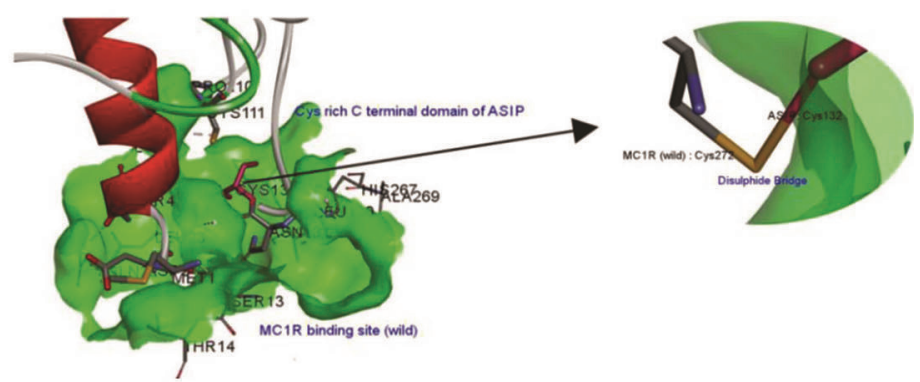

Figure 10. Interacting residues of (a) MC1R (wild) - alpha MSH complex; (b) MC1R (melanistic variant) - alpha MSH; and (c) MC1R (wild type) - ASIP complex. Enlarged: Disulphide bridge formed between Cys272 of MC1R (wild type) and Cys132 of ASIP.

In conclusion, the results of this study provide an understanding of the preferred agouti binding nature of MC1R in F. palmarum, and loss of agouti binding in the mutated variant. This does not rule out the possibility of contribution from other genes to melanism in the threestriped palm squirrel. As there are no other reports on the presence of black squirrels from the Indian subcontinent, this study has evolutionary importance as well. The observations and predictions presented here merit further research on MC1R expression and molecular dynamicsbased studies to analyse the stability of the receptor and its behaviour, in case another black squirrel is obtained from the wild.

1. McRobie, H., Moncrief, D. N. and Mundy, I. N., Multiple origins of melanism in two species of North American tree squirrel (Sciurus). BMC Evol. Biol., 2019, 19(140), 1471-1477.

2. Lin, J. and Fisher, D., Melanocyte biology and skin pigmentation. Nature, 2007, 445, 843-850.

3. D’Mello, S. A. N., Finlay, G. J., Baguley, B. C. and AskarianAmiri, M. E., Signaling pathways in melanogenesis. Int. J. Mol. Sci., 2016, 17, 1144.

4. McRobie, H., Thomas, A. and Kelly, J., The genetic basis of melanism in the gray squirrel (Sciurus carolinensis). J. Hered., 2009, 100, 709-714.

5. Almathen, F., Elbir, H., Bahbahani, H., Mwacharo, J. and Hanotte, O., Polymorphisms in MC1R and ASIP genes are associated with coat color variation in the Arabian camel. J. Hered., 2018, 6(109), 700-706.

6. Altschul, S. F., Gish, W., Miller, W., Myers, W. E. and Lipman, J. D., Basic local alignment search tool. J. Mol. Biol., 1990, 215, 403-410.

7. Kumar, S., Tamura, K. and Nei, M., MEGA: molecular evolutionary genetics analysis software for microcomputers. Comput. Appl. Biosci., 1994, 10, 189-191.

8. Ambrish, R., Alper, K. and Yang, Z., I-TASSER: a unified platform for automated protein structure and function prediction. Nature Protoc., 2010, 5, 725-738.

9. Laskoswki, R. A., MacArthur, M. W., Moss, D. S. and Thorton, J. M., PROCHECK: a program to check the stereo-chemical quality of protein structures. J. Appl. Crystallogr., 1993, 26, 283-291.

10. Hooft, R. W. W., Vriend, G., Sander, C. and Abola, E. E., Errors in protein structures. Nature, 1996, 381, 272.

11. Colovos, C. and Yeates, T. O., Verification of protein structures: patterns of non-bonded atomic interactions. Protein Sci., 1993, 2, $1511-1519$.
12. Luthy, R., Bowie, J. U. and Eisenberg, D., Assessment of protein models with three-dimensional profiles. Nature, 1992, 356, 83-85.

13. Pontius, J., Richelle, J. and Wodak, S. J., Deviations from standard atomic volumes as a quality measure for protein crystal structures. J. Mol. Biol., 1996, 264, 121-136.

14. Guex, N. and Peitsch, M. C., SWISS-MODEL and the Swiss Pdb Viewer: an environment for comparative protein modeling. Electrophoresis, 1997, 18, 2714-2723.

15. Holm, L. and Rosenstrom, P., Dali server: conservation mapping in 3D. Nucleic Acids Res., 2010, 38, W545-W549.

16. Joe, D., Zheng, O., Jeffery, T., Andrew, B., Yaron, T. and Jie, L., CASTp: computed ATAS of surface topography of proteins with structural and topographical mapping of functionally annotated residues. Nucleic Acids Res., 2006, 34, W116-W118.

17. Chen, R., Li, L. and Weng, Z. P., ZDOCK: an initial-stage protein-docking algorithm. Proteins, 2003, 52(1), 80-87.

18. Mashiach, E., Schneidman-Duhovny, D., Andrusier, N., Nussinov, R. and Wolfson, J. H., FireDock: a web server for fast interaction refinement in molecular docking. Nucleic Acids Res., 2008, 36, W229-W232.

19. Zalfa, A., Scott, C. M., Furumura, M., Lamoreux, L. M., Ollmann, M., Barsh, S. G. and Hearing, J. M., The melanocortin 1 receptor is the principal mediator of the effects of agouti signaling protein on mammalian melanocytes. J. Cell Sci., 2001, 114, 1019-1024.

20. Patel, M. P., Cribb Fabersunne, S. C., Yang, Y., Kaelin, B. C., Barsh, S. G. and Mullhauser, L. G., Loop swapped chimeras of agouti related protein (AgRP) and agouti signaling protein (ASIP) identify contacts required for melanocortin 1 receptor (MC1R) selectivity and antagonism. J. Mol. Biol., 2010, 404, 45-55.

21. McNulty, J. C. et al., Structures of the agouti signaling protein. J. Mol. Biol., 2005, 346, 1059-1070.

ACKNOWLEDGEMENTS. This work is supported by State Inter University Centre for Excellence in Bioinformatics, University of Kerala, Thiruvananthapuram. We thank Dr C. A. Jayaprakash (Scientist), V. Suresh (Field staff) and B. Jayan (Field staff) (Central Tuber Crops Research Institute, Thiruvananthapuram) for help in collecting the melanistic squirrel and the Kerala Forest Department for the necessary permissions. We also thank Mrs Jolly Sarah (Baruch, Gandhipuram, Thiruvananthapuram) for taking care of melanistic squirrel and Mrs Indira (Lab Assistant, University of Kerala) for daily monitoring the animals.

Received 14 December 2020; revised accepted 2 April 2021

doi: $10.18520 / \mathrm{cs} / \mathrm{v} 121 / \mathrm{i} 2 / 306-312$ 